\title{
The Correlation between the Degree of Enophthalmos and the Extent of Fracture in Medial Orbital Wall Fracture Left Untreated for Over Six Months: A Retrospective Analysis of 81 Cases at a Single Institution
}

\author{
Yun Sik Sung, Chan Min Chung, In Pyo Hong \\ Department of Plastic and Reconstructive Surgery, National Medical Center, Seoul, Korea
}

\begin{abstract}
Background In patients with medial orbital wall fracture, predicting the correlation between the degree of enophthalmos and the extent of fracture is essential for deciding on surgical treatment. We conducted this retrospective study to identify the correlation between the two parameters.

Methods We quantitatively analyzed the correlation between the area of the bone defect and the degree of enophthalmos on computed tomography scans in 81 patients with medial orbital wall fracture who had been left untreated for more than six months.

Results There was a significant linear positive correlation between the area of the medial orbital wall fracture and the degree of enophthalmos with a formula of $E=0.705 A+0.061(E$, the degree of enophthalmos; $A$, the area of bone defect) (Pearson's correlation coefficient, 0.812) $(P<0.05)$. In addition, that there were no cases in which the degree of enophthalmos was greater than $2 \mathrm{~mm}$ when the area of the medial orbital wall fracture was smaller than 1.90 $\mathrm{cm}^{2}$.

Conclusions Our results indicate not only that $2 \mathrm{~mm}$ of enophthalmos corresponds to a bone defect area of approximately $2.75 \mathrm{~cm}^{2}$ in patients with medial orbital wall fracture but also that the degree of enophthalmos could be quantitatively predicted based on the area of the bone defect even more than six months after trauma.
\end{abstract}

Keywords Orbital fractures / Enophthalmos / Decision support systems, clinical
Correspondence: In Pyo Hong Department of Plastic and Reconstructive Surgery, National Medical Center, 245 Eulji-ro, Jung-gu, Seoul 100-799, Korea

Tel: $+82-2-2260-7207$

Fax: +82-2-2272-7207

E-mail:nmcps@unitel.co.kr

No potential conflict of interest relevant to this article was reported.

Received: 14 Mar 2013• Revised: 26 May $2013 \bullet$ Accepted: 11 Jun 2013

pISSN: 2234-6163 • elSSN: 2234-6171 • http://dx.doi.org/10.5999/aps.2013.40.4.335• Arch Plast Surg 2013;40:335-340

\section{INTRODUCTION}

Controversy remains over the treatment of orbital wall fracture. In some cases of fracture, there is no need for treatment, in other cases surgical intervention is essential. According to Converse and Smith [1], clinicians should perform an early repair of the defect to prevent the occurrence of functional impairment. In addition, Bowers [2] reported that clinicians should decide on surgical interventions through the careful clinical examination of such conditions as diplopia, restricted gaze field, and enoph-

Copyright $($ C 2013 The Korean Society of Plastic and Reconstructive Surgeons

This is an Open Access article distributed under the terms of the Creative Commons Attribution Non-Commercial License (http://creativecommons.org/

licenses/by-nc/3.0/ which permits unrestricted non-commercial use, distribution, and reproduction in any medium, provided the original work is properly cited.

www.e-aps.org 
thalmos. In an actual clinical setting, diplopia, enophthalmos, or restriction of gaze are often used to determine the necessity for repair of an orbital wall fracture [3,4]. However, diplopia and restriction of eye motility may be temporary findings that occur as a result of swelling, contusion, or intramuscular hematoma, and can resolve with time. The degree of enophthalmos is the most valuable basis for deciding on surgical reconstruction of orbital wall fracture [5]. Specifically, it has been reported that the degree of enophthalmos of $2 \mathrm{~mm}$ is an indicator of achieving satisfactory cosmetic results. Migliori and Gladstone [6], and Koo et al. [7] reported that no visual impairment occurred when the projection of the two eyeballs differed by $<2 \mathrm{~mm}$. Most of the studies in this series have also shown that surgical treatment is indicated for patients with an enophthalmos of $>2 \mathrm{~mm}$ [8]. It should be noted, however, that the enophthalmos may not be present immediately after the onset of trauma. It cannot be easily detected in the early posttraumatic period because of orbital edema [9].

It has been reported that the degree of enophthalmos was higher in patients with a greater extent of orbital wall fracture. In addition, there was a significant correlation between the two factors [10].

A Hertel exophthalmometer is a standard method for documenting the degree of exophthalmos, but it shows low reliability, poor reproducibility, and measurement errors depending on the investigator as well as the ocular status of the patients [11]. Therefore, computed tomography (CT) scans are used for the analysis of orbital fracture.

We have speculated that if we can predict the degree of enophthalmos in the early stage of the onset of trauma by analyzing its correlation with the extent of fracture, this would be of great help for planning treatment as early as possible.

To date, many attempts have been made to clarify the correlation between the degree of enophthalmos and the area of the bone defect. To our knowledge, however, there are no reports in patients who had been left untreated for more than six months.

Given the above background, we conducted this retrospective study to identify the correlation between the degree of enophthalmos and the extent of fracture in 81 patients who had been left untreated for more than six months.

\section{METHODS}

\section{Study patients}

Of the patients who visited the emergency room of our medical institution during a period ranging from 2009 to 2012, 81 had medial orbital wall fracture detected incidentally.

The inclusion criteria for the current study were as follows: 1)
Patients with medial orbital wall fracture who underwent facial CT examination for the evaluation of other symptoms than the trauma of the region adjacent to the orbit, 2) Patients who had no history of sustaining additional trauma to the region adjacent to the orbit or undergoing any other treatment for craniofacial injury within the most recent six months before the date of CT examination, 3) Patients who presented with no zygomaticomaxillary fracture, 4) Patients who had no deviation of the nasal septum, 5) Patients who had no nasal bone fracture, 6) Patients who had no bilateral orbital bone fractures, and 7) Patients whose eyeballs had identical vertical lengths.

In addition, in patients with ocular regression or a discrepancy bilaterally in the vertical length of the eyeball, we could not compare the degree of enophthalmos between the two sides of the orbit. The corresponding cases were therefore excluded from the current analysis.

The current study was approved by the Institutional Review Board of our medical institution and because of its retrospective nature, the requirement for obtaining a written informed consent was waived.

\section{Study methods}

In the current study, we performed a CT scan of the medial orbital wall fracture and thereby analyzed the extent of fracture (the area of bone defect) at a thickness of $2.5 \mathrm{~mm}$ based on the non-overlapping, non-serial sections. In addition, we also measured the area of bone defects and the degree of enophthalmos using the PiView star (Infinitt Co. Ltd., Seoul, Korea), a picturearchiving and communication system (PACS).

\section{Fig. 1. The measurement of bone defect areas}

To calculate the area of the bone defect, we set X (the length of the medial wall defect) and $A$ (the area of bone defects in each section = $X \times 2.5 \mathrm{~mm}$ [slice thickness]). Then, we used a formula $\sum_{n=1}^{N} A_{n}$ to sum the areas of the bone defects in each section.

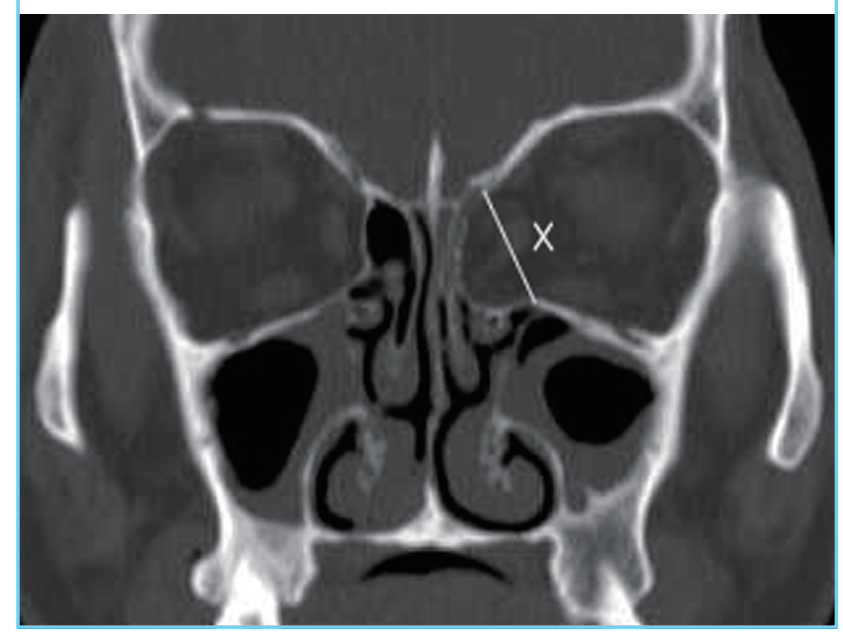


The measurement of the bone defect area at the medial orbital wall fracture site

As shown in Fig. 1, based on the symmetric features of the bilateral orbital bones, we measured the length of the line extending from the starting point of the medial orbital wall fracture to its end using the PACS at each section on coronal CT scans and designated it as $X$. We multiplied it by the interval of CT scanning and thereby obtained the unit area of the bone defect. By summing the measurements of each area, we calculated the total area of the bone defect and designated it as $A$. It is probable that the area measured could have been greater than the actual value. However, we selected this method because it can be easily used in clinical examinations.

The measurement and comparison of the degree of enophthalmos between the two sides

Solely on transverse CT scans that were taken on frontal gaze, clearly visualizing the center of both eyeballs and maximizing the size of both eyeballs containing the corneal apex, we measured the location of the eyeball. The distance extending between the two lateral orbital rims on $\mathrm{CT}$ scans was defined as line $\mathrm{A}$. In addition, the shortest distance extending from the corneal center to line $\mathrm{A}$ was defined as line $\mathrm{B}$, while the line extending between the lateral orbital rim and the medial orbital rim was defined as line C. Finally, the line extending from the corneal apex to line $\mathrm{C}$ via the center of the eyeball was defined as line $\mathrm{D}$ [11]. All of the parameters are schematically illustrated in Fig. 2. The globe position was measured from the difference in the value of $\mathrm{D}$. The enophthalmos was defined as the difference

\section{Fig. 2. Parameters for the degree of enophthalmos}

To measure the degree of enophthalmos, we defined the distances $A$, the distance between the lateral orbital rims of both eyes; $B$, the shortest distance extending from the corneal center to line $A ; C$, the distance between the lateral and medial orbital margins; and $D$, the length of the line passing through the corneal center from its apex to line $\mathrm{C}$.

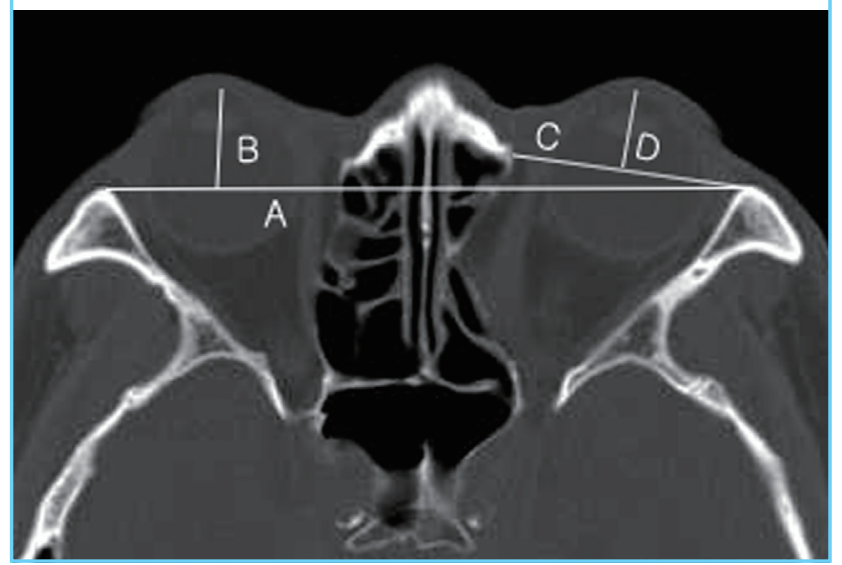

in the distance between the normal eye and the traumatized one. However, we did not use the difference in the value of $B$ in measuring the globe position because if there was a discrepancy in the visual axis of the eye, this would cause measurement errors and then make it difficult to determine the degree of enophthalmos.

\section{Statistical analysis}

All measurements were expressed as mean \pm standard deviation. Statistical analysis was done using SPSS ver. 12.0 for Windows (SPSS Inc., Chicago, IL, USA). To identify the correlation between the degree of enophthalmos and the area of the bone defect, we performed a Pearson's correlation analysis. A P-value of $<0.05$ was considered statistically significant.

\section{RESULTS}

\section{Baseline and clinical characteristics of the patients}

The patients had a mean age of $50.2 \pm 12.3$ years (range, 24 to 76 years). They consisted of 75 men (92.6\%) and 6 women (7.4\%), showing a male predilection. Moreover, there were 31 right-sided cases (38.3\%) and 50 left-sided ones (61.7\%).

\section{The correlation between the degree of enophthalmos and the area of bone defect}

Following a retrospective analysis of a consecutive series of 81

\section{Fig. 3. The bone defect and enophthalmos}

A regression analysis showed that a bone defect area of $2.75 \mathrm{~cm}^{2}$ corresponded to $2 \mathrm{~mm}$ of enophthalmos based on a formula of $E=0.705 A+0.061(E$, the degree of enophthalmos; $A$, the area of bone defect).

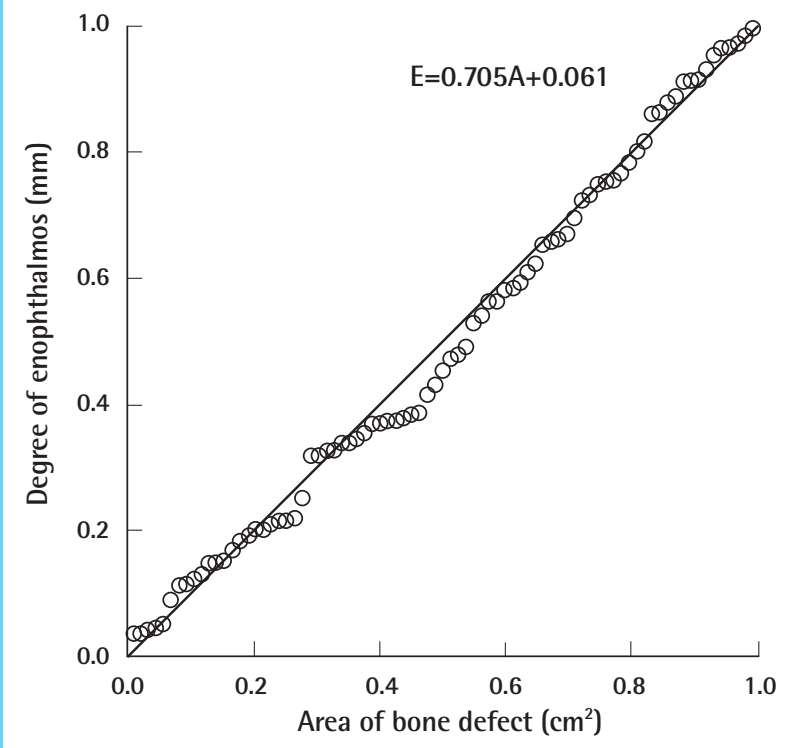


patients, there was a significant positive linear correlation between the area of the medial orbital wall fracture and the degree of enophthalmos with a formula of $\mathrm{E}=0.705 \mathrm{~A}+0.061$ (E, the degree of enophthalmos; $\mathrm{A}$, the area of the bone defect) (Pearson's correlation coefficient, $0.812 ; \mathrm{P}<0.05$ ). In addition, there were no cases in which the degree of enophthalmos was higher than $2 \mathrm{~mm}$ when the area of the medial orbital wall fracture was smaller than $1.90 \mathrm{~cm}^{2}$ (Fig. 3).

\section{DISCUSSION}

With recent technological advancements in diagnostic imaging modalities, orbital wall fracture has been detected with increased frequencies [12-14]. Treatment methods for the orbital fracture can be divided into conservative treatment and surgery. In recent years, many studies have reported that good treatment outcomes from both functional and aesthetic perspectives are also achieved with the sole use of conservative treatment $[15,16]$. This demonstrates that precise recommendations the surgical management of orbital bone fracture should be followed.

The presence of diplopia, enophthalmos, or restriction of gaze are often used as criteria for repair of fractures of the orbital wall $[3,4]$. Enophthalmos rarely occurs in the early stage of trauma, but it is the most valuable indicator for deciding on surgical reconstruction of the orbital wall fracture. Diplopia and restriction of eye motility may be temporary findings that occur as a result of swelling, contusion, or intramuscular hematoma, both of which will resolve with time. However, there is a lack of valid diagnostic criteria for predicting whether orbital wall fracture may result in functional impairment.

According to several studies, quantifying the area of the bone defect on CT scans in patients with orbital wall fracture is essential for deciding on a treatment approach and is often used for deciding whether to perform surgery. Attempts have also been made to identify the correlation of enophthalmos with such factors as diplopia, eye motility, and the size of the bone fracture. It has been shown, however, that the degree of enophthalmos only had a correlation with the size of the bone fracture [10]. In an actual clinical setting, however, it is not easy to collect data from many cases of orbital fracture that have been left untreated for long periods of time.

In the current study, we examined medial orbital wall fracture because inferior orbital wall fracture is more often complicated by diplopia, ocular disorder, sensory disturbance, and compound fracture than is medial orbital wall fracture. Accordingly, it is less probable that clinicians would decide on surgical treatment solely based on the area of the bone defect in cases of me- dial orbital wall fracture.

In addition, we also found that a significantly larger number of patients with medial orbital wall fracture had been left untreated than the number of those with inferior orbital wall fracture. Presumably, this might be for the following reasons: 1) The entrapment of the extraocular muscle between the fractures is less frequently seen in medial orbital wall fracture in inferior orbital wall fracture. 2) It is easier for patients to adapt themselves to daily life when dealing with horizontal diplopia from medial orbital wall fracture than with vertical diplopia from inferior orbital wall fracture. 3) As mentioned earlier, medial orbital wall fracture is less frequently complicated by sensory disturbance and combined fracture than is inferior orbital wall fracture. This lowers the frequency of early surgical treatment.

Previous studies have reported that the degree of enophthalmos can be predicted to be approximately $2 \mathrm{~mm}$ when the area of the bone defect is $1.9 \mathrm{~cm}^{2}$ in cases of medial orbital wall fracture $[10,17]$. However, these studies' reliability may be somewhat lower because they enrolled a small number of patients and measured the difference in the location of the eyeballs using a Hertel exophthalmometer. In the current study, however, we measured the difference in the location of the eyeballs on CT scans because Hertel exophthalmometry has been reported by some to be rather unreliable due to factors associated with the patients or investigators.

Patient-related factors include any asymmetry of the facial skeleton. A Hertel exophthalmometer can produce measurement errors when its foot plate is incorrectly positioned on the lateral orbital rim. Since it is placed on soft tissue, the position of the reference point is subject to change depending on the investigator. The degree of compression is another factor that may influence the results of Hertel exophthalmometry $[11,18]$.

For these reasons, a Hertel exophthalmometer is a non-invasive, simple modality, but its reliability and reproducibility are relatively low. Measuring the location of the eyeball on CT scans would decrease the measurement error.

We therefore used CT to examine the relationship between the bone defect area and enophthalmos. In addition, because patients do not undergo CT scanning in a frontal gaze in an actual clinical setting, we defined the value of line $\mathrm{D}$ to adjust this. Then, to ensure that the axial length of the globe remains constant, we performed radiologic examinations using orbital CT computed tomography. Thus, we attempted to obtain reliable measurements of the degree of enophthalmos useful for clinical application.

In a clinical setting, clinicians are routinely required to measure the area of a bone defect and thereby to decide on the appropriate surgical treatment and approach. Some clinicians also use a 
formula of $\pi \times A B / 4$ (A, the height of the medial wall defect on coronal views; $\mathrm{B}$, the length of the medial wall defect on axial views) by calculating the values of the long and short axis of the bone defect of the orbit on CT scans $[10,19,20]$. However, this formula is valid only when the fracture has an elliptical shape. If the fracture has an irregular shape, the measurement error would be further increased. In the current study, we summed the areas of each section using the PACS. It is also probable that this simple integral method may increase the size of measurements as compared with the actual values of the area of the bone defect. However, because it is easy to use and can produce results that are the closest to the actual values, we used the above method for the current study.

To summarize, our results showed a correlation between the area of the medial bone defect and the degree of enophthalmos with a formula of $\mathrm{E}=0.705 \mathrm{~A}+0.061$ ( $\mathrm{E}$, the degree of enophthalmos; and A, the area of bone defect), based on which, we can speculate that the degree of enophathlmos of $2 \mathrm{~mm}$ would correspond to a bone defect area of approximately $2.75 \mathrm{~cm}^{2}$ when considering surgical treatment of patients.

Our results also indicate that the degree of enophthalmos could be quantitatively predicted based on the area of the bone defect even more than six months after the onset of trauma. This will be of help for clinicians in deciding on surgical treatment for patients with medial orbital wall fracture who did not undergo early treatment. Nevertheless, the current data has been collected from a long-term follow-up period of longer than six months. It should therefore be noted that in addition to the area of the bone defect, the discrepancy in the location of the eyeball may also arise from such factors as fat atrophy. Moreover, further studies are also warranted to develop more reliable methods for measuring each parameter.

Based on our results, we propose the following treatment strategy: 1) Surgery should be immediately performed in patients with an area of bone defect of $>2.75 \mathrm{~cm}^{2} .2$ ) The clinical course should be monitored in patients with an area of bone defect of 1.90 to $2.75 \mathrm{~cm}^{2}$. Even in these patients, however, surgery should be considered if the degree of enophthalmos exceeded 2 $\mathrm{mm}$ during the follow-up period. 3) Conservative treatment is sufficient in patients with an area of bone defect of $<1.90 \mathrm{~cm}^{2}$.

\section{REFERENCES}

1. Converse JM, Smith B. Enophthalmos and diplopia in fractures of the orbital floor. BrJ Plast Surg 1957;9:265-74.

2. Bowers JF. The management of blow-out fracture of the orbital floor. Surv Ophthalmol 1964;40:237-43.

3. Joseph JM, Glavas IP. Orbital fractures: a review. Clin Oph- thalmol 2011;5:95-100.

4. Mathog RH. Management of orbital blow-out fractures. Otolaryngol Clin North Am 1991;24:79-91.

5. Kunz C, Sigron GR, Jaquiery C. Functional outcome after non-surgical management of orbital fractures-the bias of decision-making according to size of defect: critical review of 48 patients. Br J Oral Maxillofac Surg 2012 Nov 7 [Epub]. http://dx.doi.org/10.1016/j.bjoms.2012.09.016.

6. Migliori ME, Gladstone GJ. Determination of the normal range of exophthalmometric values for black and white adults. Am J Ophthalmol 1984;98:438-42.

7. Koo L, Hatton MP, Rubin PA. When is enophthalmos "significant”? Ophthal Plast Reconstr Surg 2006;22:274-7.

8. Osguthorpe JD. Orbital wall fractures: evaluation and management. Otolaryngol Head Neck Surg 1991;105:702-7.

9. Kim YK, Park CS, Kim HK, et al. Correlation between changes of medial rectus muscle section and enophthalmos in patients with medial orbital wall fracture. J Plast Reconstr Aesthet Surg 2009;62:1379-83.

10. Jin HR, Shin SO, Choo MJ, et al. Relationship between the extent of fracture and the degree of enophthalmos in isolated blowout fractures of the medial orbital wall. J Oral Maxillofac Surg 2000;58:617-20.

11. Nkenke E, Maier T, Benz M, et al. Hertel exophthalmometry versus computed tomography and optical 3D imaging for the determination of the globe position in zygomatic fractures. Int J Oral Maxillofac Surg 2004;33:125-33.

12. Kim IT, Choi JB. Normal range of exophthalmos values on orbit computerized tomography in Koreans. Ophthalmologica 2001;215:156-62.

13. Tanaka T, Morimoto Y, Kito S, et al. Evaluation of coronal CT findings of rare cases of isolated medial orbital wall blow-out fractures. Dentomaxillofac Radiol 2003;32:300-3.

14. Caranci F, Cicala D, Cappabianca S, et al. Orbital fractures: role of imaging. Semin Ultrasound CT MR 2012;33:38591.

15. Kim HW, Kim YI, Won IK. Clinical analysis of blow-out fracture with ocualr motion limitation: comparison of surgical and conservative treatment. J Korean Ophthalmol Soc 1999;40:632-8.

16. Kim SK, Chang HK. The clinical study of treatment of blowout fracture. J Korean Ophthalmol Soc 1995;36:162935.

17. Snell BJ, Flapper W, Moore M, et al. Management of isolated fractures of the medial orbital wall. J Craniofac Surg 2013;24:291-4.

18. Davanger M. Principles and sources of error in exophthalmometry. A new exophthalmometer. Acta Ophthalmol 
(Copenh) 1970;48:625-33.

19. Manson PN, Grivas A, Rosenbaum A, et al. Studies on enophthalmos: II. The measurement of orbital injuries and their treatment by quantitative computed tomography. Plast
Reconstr Surg 1986;77:203-14.

20. Hwang K, Huan F, Hwang PJ. Diplopia and enophthalmos in blowout fractures. J Craniofac Surg 2012;23:1077-82. 